\title{
Can be tracing of surgeon's hand movement useful in laparoscopic and endoscopic learning curve?
}

\author{
Cambal M, Labas P, Lukac L, Skoda A, Zemanova M \\ 1st Department of Surgery, School of Medicine, Comenius University in Bratislava, University Hospital Bratislava, Slovakia.
}

\begin{abstract}
Minimally invasive surgical and endoscopic procedures in modern medicine have took a stable place and became a gold standard in the diagnosis and treatment of many diseases. Unlike conventional surgical procedures, these techniques require more than just surgical workplace equipment and trained doctors, capable to perform minimally invasive procedures. Opposed to „open“ procedures requires a relatively long acquisition of skills and training called „learning curve“. One of the ways to acquire this skills, in addition to the continuous improvement of surgical techniques during surgical procedures training on the laparoscopic simulator. Modern laparoscopic and endoscopic simulators are furnished except simulations of various operations to analyze the trajectory of motion used instruments and on the basis of the evaluation to assess the inclusion of physician skills in laparoscopy / endoscopy. Another view on the issue of the effectiveness of movements addressed at the 1st. Departement of Surgery (Medical School, Comenius University in Bratislava) - assess the trajectory of hand movements of the surgeon and endoscopist during the execution of a procedure not only in the simulator, but also during laparoscopic / endoscopic surgery.

Within the project „Applied research trajectory hands in laparoscopic and endoscopic operations“, ITMS Project code 26240220056, funded by the European Regional Development Fund and the state budget of the Slovak Republic, we created a
\end{abstract}

technical background and algorithms for monitoring and evaluating the hand movements of the specialist physician during endoscopic procedures. This is a unique idea and unique project transformed into clinical practice, which is promising to assist in endoscopy training and inclusion endoscopist to „skilfulness“ group on the evaluation of the effectiveness of movements of his hands.

The aims of the project are the options of universal application assessing the skills in the laparoscopic and endoscopic surgeries.

Based on theoretical and applied research it was able to test the chosen participants with various skills and abilities in the endoscopic surgeries, resulted with their categorization into predefined skill categories, based on the trajectories of hand movement.

The basic idea, which in any case can not be ignored, not only in the solved problem, but in medicine generally, remains the safety of laparoscopic / endoscopic intervention for patient, regardless of the individual differences in the technique of particular surgeons or to time differences in the performance of different types of operations.

Important impact of the proposed application utilization may also be a draft modification to operational processes and tool, and their use in terms of ergonomics pursued practices.

The new method brings not only new opportunities but also new insights into the surgery, which was once „a matter of golden hands of a surgeon with a scalpel“. 